\title{
Pain in Portuguese patients with multiple sclerosis
}

\author{
Daniela Seixas ${ }^{1,2,3 *}$, Maria José Sá ${ }^{4,5}$, Vasco Galhardo ${ }^{1,2}$, Joana Guimarães ${ }^{4,6}$ and Deolinda Lima ${ }^{1,2}$ \\ 1 Institute of Histology and Embryology, Faculty of Medicine of Porto University, Porto, Portugal \\ 2 Instituto de Biologia Molecular e Celular, Porto, Portugal \\ ${ }^{3}$ Department of Imaging, Centro Hospitalar de Vila Nova de Gaia/Espinho, Porto, Portugal \\ ${ }^{4}$ Department of Neurology, Hospital de São João, Porto, Portugal \\ ${ }^{5}$ Health Sciences Faculty, University Fernando Pessoa, Porto, Portugal \\ ${ }^{6}$ Department of Neurology and Neurosurgery, Faculty of Medicine of Porto University, Porto, Portugal
}

\section{Edited by:}

Hans-Peter Hartung,

Heinrich-Heine-Universität

Düsseldorf, Germany

\section{Reviewed by:}

Ludwig Kappos, University Hospital

Basel, Switzerland

Matthew Mccoyd, Loyola University

Hospital, USA

*Correspondence:

Daniela Seixas, Instituto de Histologia e Embriologia, Faculdade de Medicina da Universidade do Porto, Alameda Prof. Hernâni Monteiro, 4200, 319

Porto, Portugal.

e-mail:dseixas@med.up.pt

\begin{abstract}
Early reports often ignored pain as an important symptom in multiple sclerosis (MS). Pain prevalence figures in MS from European countries other than Portugal range between 40 and $65 \%$. To our knowledge there is no published data in English on pain in MS in Portugal. We describe the demographic and clinical characteristics, with an emphasis on pain, of 85 MS patients followed-up in a Portuguese hospital, contributing to pain epidemiology in MS. Patients were interviewed sequentially after their regular appointments at the MS clinic; patients with pain completed The Brief Pain Inventory and The McGill Pain Questionnaire (MPQ). The prevalence of pain found was $34 \%$. Headache and back pain were the most common anatomical sites described, followed by upper and lower limbs. Intensity of pain in an 11-point scale was, for the maximum pain intensity $6.7 \pm 1.8$, for the minimum pain intensity $2.2 \pm 2.0$, for the mean pain intensity $4.5 \pm 1.5$, and for the actual pain intensity $2.4 \pm 2$.9. Pain interfered significantly with general activity, mood, work, social relations, and enjoyment of life. All MS patients with pain employed words from both the sensory and affective categories of the MPQ to describe it. Patient pain descriptions' included the word "hot-burning" in 59\% of the cases, common in the report of central pain, but neuropathic pain medications were only used by $10 \%$ of them. Pain is an important symptom in Portuguese patients with MS, not only because of the high prevalence found, concordant with other European countries, but also because of its interference with quality-of-life.
\end{abstract}

Keywords: multiple sclerosis, pain, quality-of-life, epidemiology, Europe

\section{INTRODUCTION}

Early reports stated that pain was an uncommon symptom in multiple sclerosis (MS), and during the 1980s it was not valued among the constellation of MS clinical problems (Tourtellote and Baumhefner, 1983). However, it soon became evident that many patients with MS suffer from persistent pain during the course of their disease. Results from European countries other than Portugal (namely, Italy, Belgium, and Denmark) showed pain prevalence figures in MS ranging between 40 and 65\% (Vermote et al., 1986; Stenager et al., 1991; Boneschi et al., 2008), and pain has been recognized as important in the overall health-related quality-of-life (QoL) of MS patients (Svendsen et al., 2005). Solaro et al. (2004) in a cross-sectional study that involved 1672 Italian patients further explored pain frequencies in MS reporting a prevalence of trigeminal neuralgia of $2 \%$, Lhermitte's sign $9 \%$, dysesthetic pain $18.1 \%$, back pain $16.4 \%$ and painful tonic spasms $11 \%$. In the United States of America and Canada, pain in MS was reported in 66 and $46 \%$ of patients, respectively (Ehde et al., 2006; Piwko et al., 2007). Pain severity in MS was identified in some studies to be associated with female sex, increased age, lower educational level, non-stable MS disease course, longer duration of pain, depression, increased disability, and greater health care use (Hadjimichael et al., 2007). Ultimately, a better knowledge about pain characteristics in MS will lead to improved care and treatment.
In the 1980s the Iberian Peninsula, comprising Portugal and Spain, was classified as a low-medium frequency zone for MS (Kurtzke, 1980). However, current data has revealed otherwise (Rosati, 2001), and a recent study has shown a MS prevalence of 46.3 per 100000 in a region of Portugal (De Sá et al., 2006). Given the particularities of the disease, all MS patients in Portugal are followed up in specialized MS clinics managed in the largest tertiary hospitals. The MS clinic of São João Hospital assists 600 patients per year, $10 \%$ of which are newly diagnosed MS cases; these patients come from the northern part of the country, which has roughly three million inhabitants.

The objective of this study is to characterize Portuguese MS patients using two tests of pain measure descriptors, and comparing these results with data from other countries. To our knowledge, this is the first study that has examined pain in Portuguese MS patients.

\section{MATERIALS AND METHODS}

Eighty-five patients from the universe of patients of the MS clinic of São João Hospital, Porto, Portugal, participated in the study. To be included, the patients had to be more than 18 years old and have a definitive diagnosis of MS according to the revised McDonald criteria (Polman et al., 2005). Exclusion criterion was serious cognitive impairment. Patients in follow-up at the clinic were 
identified and recruited consecutively during the period of 1 year (2007), after their regular appointments. Eight patients declined to enter the study (not willing to participate), and four subjects were excluded based on the study criterion. The project was approved by the São João Hospital Ethics Committee; informed consent was obtained from all subjects.

The clinical records of each patient were consulted to obtain data on age, gender, education level, race/ethnicity, disease duration, year of diagnosis, MS type (explained ahead), treatments, and the presence of pain. The course of MS develops broadly into four disease types: relapsing-remitting, secondary progressive, progressive-relapsing, and primary progressive. The clinical evolution of MS usually occurs in relapses in the first years of the disease, with remission of the symptoms and signs (relapsingremitting), and then becoming progressive with time (secondary progressive MS). Other subtypes of the disease, more aggressive, are progressive-relapsing MS (the signs and symptoms of the disease do not abate completely after each relapse), and primary progressive MS that lacks the characteristic episodic evolution, being progressive from the onset. Additionally, the first symptomatic form of the disease is the denominated clinically isolated syndrome.

For each patient the score of the Expanded Disability Status Scale (EDSS; Kurtzke, 1983) was determined by a neurologist on the day of the study session. The short version of the pain questionnaires The Brief Pain Inventory (BPI; Daut et al., 1983) and The McGill Pain Questionnaire (MPQ; Melzack, 1987) were administered to patients who reported pain during the week previous to the study session.

\section{EXPANDED DISABILITY STATUS SCALE}

The EDSS is a method of quantifying disability in MS. The score is based on neurological testing of eight functional systems: pyramidal, cerebellar, brainstem, sensory, bowel and bladder, visual, mental, and other (Kurtzke, 1983). The EDSS has a possible range from 0 , indicating no disability and normal neurological examination, to 10, referring to death due to MS. EDSS steps 1.0-3.5 refer to patients with MS that are fully ambulatory; EDSS steps 4.0-9.5 are defined by the impairment to ambulation. In the present study EDSS scores were categorized as mild (0-3.5), moderate (4.0-6.0), and severe (6.5-9.5).

\section{SHORT VERSION OF THE BRIEF PAIN INVENTORY}

The BPI was developed to evaluate the intensity and impact of pain in the daily functioning of patients. Osborne et al. (2006) evaluated the psychometric properties of the short version of the BPI in patients with MS and pain, and concluded that results support the validity of the modified versions of the original long form of the BPI. The short version of the BPI consists of a diagram to record pain location in a human figure and scales for pain intensity and impact evaluation. Pain intensity is registered in numerical scales that vary from zero ("no pain") to 10 ("the worst pain possible"). Impact of pain is measured in relation to how the pain interferes with general activity, mood, walking ability, work, social relations, sleep, and enjoyment of life in 11-point scales ("no interference" to "interferes completely"). Patients are asked as well about their pain treatment and relief obtained with it.

\section{SHORT VERSION OF THE McGill PAIN QUESTIONNAIRE}

The short version of the MPQ evaluates pain dimensions not measured in BPI by estimating the sensory and affective elements of pain, both qualitatively and quantitatively (Melzack, 1987). It consists of 15 words, 11 representing the sensory category of pain and four representing the affective category of pain, taken from the long version of the MPQ. Detailed description of the long version of the MPQ was reported elsewhere (Melzack, 2005). Pain intensity is classified for each word contained in the short version of the MPQ (see Table 3 for a list of words) using a fourpoint scale $(0=$ "no pain", $1=$ "mild pain", $2=$ "moderate pain", 3 = "high pain"). Two scores are obtained from the sum of the sensory and the affective ratings; the total score is obtained adding the sensory and affective scores. The short version of the MPQ also includes a measure for current pain intensity score, which is a six-point scale from $0=$ "no pain", 1 = "mild", 2 = "discomforting", $3=$ "distressing", $4=$ "horrible" to $5=$ "excruciating", and a visual analogue scale.

\section{STATISTICAL ANALYSIS}

There was no missing data. Distributions by frequency were generated for every categorical variable for the descriptive aspects of the analysis. For homogenous variances, the $t$ test of Student was used to compare two patient groups (pain and no pain). For non-homogenous variances, the Mann-Whitney test was adopted. The Chi-square test was used for comparisons between categorical variables. Relationships between age, disease duration, neurologic symptom severity (EDSS) and pain intensity were assessed using Spearman or Pearson correlation coefficients as appropriate. Statistical significance was considered for $p$ values inferior to 0.05 . Statistical analyses were performed using SPSS (SPSS software INC) and results are presented as mean respectively median \pm SD.

\section{RESULTS}

The demographical characteristics of the MS patient sample (Table 1) shows that most of the patients were young adults, predominantly females. The education level was low, and the authors noted reading literacy problems during the questionnaires' filling by the patients.

Considering the clinical characteristics of the patient sample (Table 2), median EDSS score was 2. The majority of patients suffered from the relapsing-remitting subtype of the disease, and the overall distribution of disease characteristics' was similar to that described for other European MS patient populations (Alonso and Hernán, 2008). Eleven percent of the cases were clinically isolated syndromes, with clinical, laboratorial, and magnetic resonance imaging results that allowed for MS diagnosis.

Twenty-nine patients of a total of $85(34 \%)$ had pain in the day and/or the week previous to the study session. Six patients $(2 \%)$ reported pain in more than one location. Comparing the group of MS patients with pain with the group of MS patients without pain, no difference was found pertaining gender (Chisquare, $p=0.672$ ), mean age ( $t$ test, $p=0.371)$, EDSS score by categories (Chi-square, $p=0.995$ ), mean duration of the disease (in years; Mann-Whitney, $p=0.771)$, use of disease-modifying therapy (Chi-square, $p=0.680$ ), or mean education in years ( $t$ test, 
Table 1| Demographic characteristics of the MS patient sample.

\begin{tabular}{ll}
\hline & Mean (SD, min, max) \\
\hline Age & $39.7(11.7,18,64)$ \\
& Number of patients (\%) \\
Gender & \\
Male & $23(27)$ \\
Female & $62(73)$ \\
Education level & \\
4 years or less & $22(26)$ \\
6 years & $14(17)$ \\
9 years & $9(11)$ \\
12 years & $23(27)$ \\
More than 12 years & $17(20)$ \\
Race/ethnicity & \\
Caucasian & $85(100)$ \\
Marital status & \\
Married or living as married & $54(63)$ \\
Single & $21(25)$ \\
Divorced & $9(11)$ \\
Widowed & $1(1)$ \\
\hline
\end{tabular}

Table 2 | Clinical characteristics of the MS patient sample.

\begin{tabular}{ll}
\hline & Mean (SD) \\
\hline Disease duration in years & $10.9(7.1)$ \\
Years since the diagnosis & $11.3(9.7)$ \\
& Median \\
EDSS score & 2 \\
& Number of patients (\%) \\
MS type & \\
Relapsing-remitting & $60(71)$ \\
Secondary progressive & $15(18)$ \\
Primary progressive & $1(1)$ \\
Clinically isolated syndrome & $9(11)$
\end{tabular}

Table 3 | Qualitative pain characteristics: list of words representative of the sensory and affective dimensions of the short version of The McGill Pain Questionnaire, and percentage of MS patients with pain that used each word.

\begin{tabular}{ll}
$\begin{array}{l}\text { Sensory dimension } \\
\text { (\% of patients) }\end{array}$ & $\begin{array}{l}\text { Affective dimension } \\
\text { (\% of patients) }\end{array}$ \\
\hline Throbbing (0) & Tiring-exhausting (94.1) \\
Shooting (47.1) & Sickening (85.4) \\
Stabbing (70.6) & Fearful (52.9) \\
Sharp (64.7) & Cruel-punishing (23.5) \\
Cramping (0) & \\
Gnawing (41.2) & \\
Hot-burning (58.8) & \\
Aching (64.7) & \\
Heavy (94.1) & \\
Tender (47.1) & \\
Splitting (41.2)
\end{tabular}

$p=0.241)$. Age, disease duration or neurologic symptom severity (EDSS) were not significantly correlated with pain intensity ( $r=0.218, r=0.321, r=0.092, p>0.05$, respectively).

Twenty-one patients (72\%), of the total of 29 patients with pain in our study, completed the short versions of the pain questionnaires BPI and MPQ. Considering the results of the BPI on pain localization, shown in Figure 1, headache and back pain were the most common anatomical sites described (27\%), followed by upper (20\%) and lower limbs (13\%). Experienced pain was more often superficial (86\%) as opposed to deep (14\%). Pertaining to intensity of pain in an 11-point scale (Figure 2), it was, for the maximum pain intensity $6.7 \pm 1.8$, for the minimum pain intensity $2.2 \pm 2.0$, for the mean pain intensity $4.5 \pm 1.5$ and for the actual pain intensity $2.4 \pm 2.9$. Sixty-two percent of the patients were taking pain medications - with a mean relief of $75 \%$ $( \pm 28)$ - and $10 \%$ were being treated with drugs for neuropathic pain (gabapentin, amytriptiline, carbamazepine). The remainder were being treated with analgesics (paracetamol, caffeine) and/or non-steroidal anti-inflammatory drugs. Interference of pain (in a scale from 0 to 10 points) with general activity was $6.3 \pm 4.1$, with

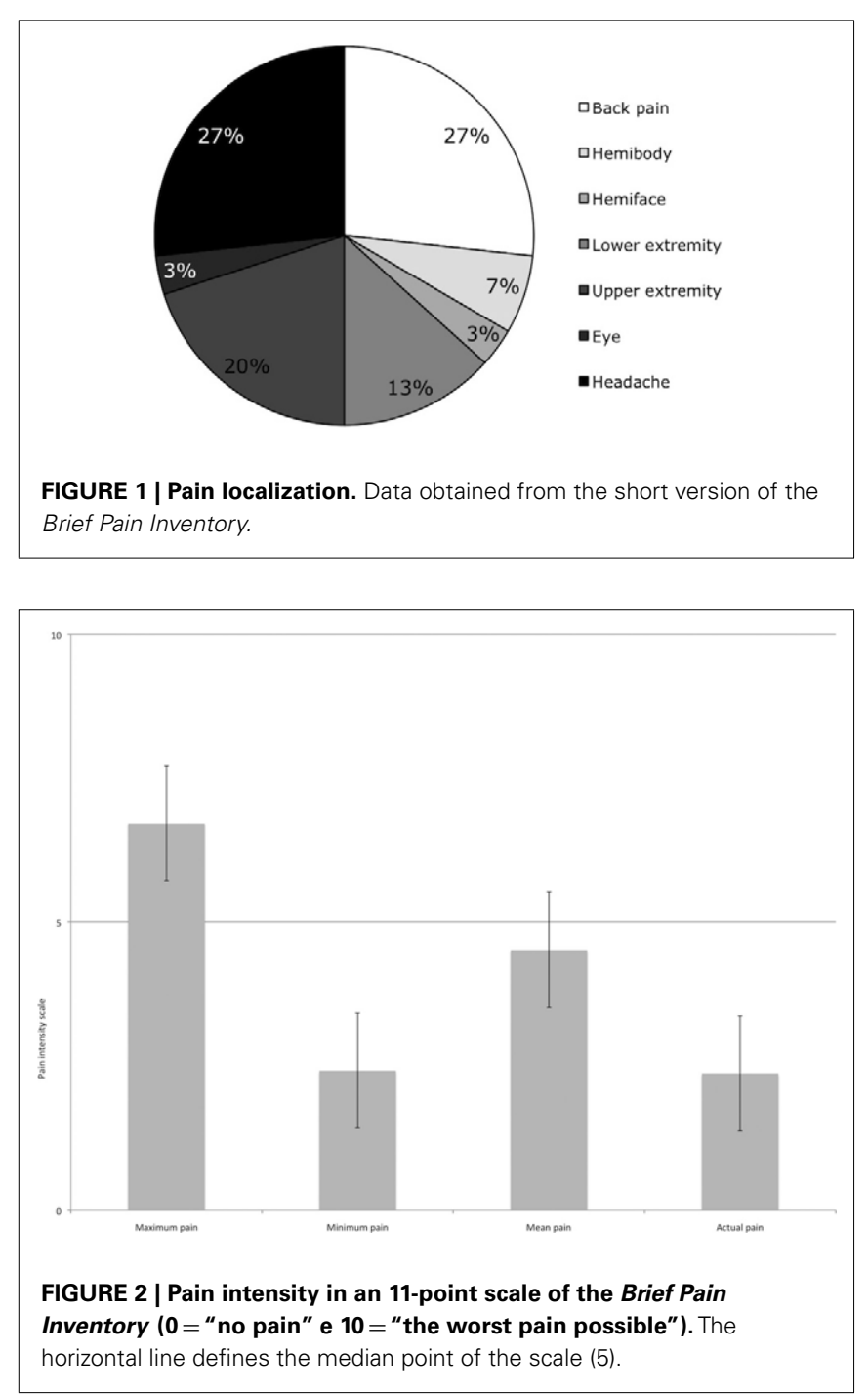




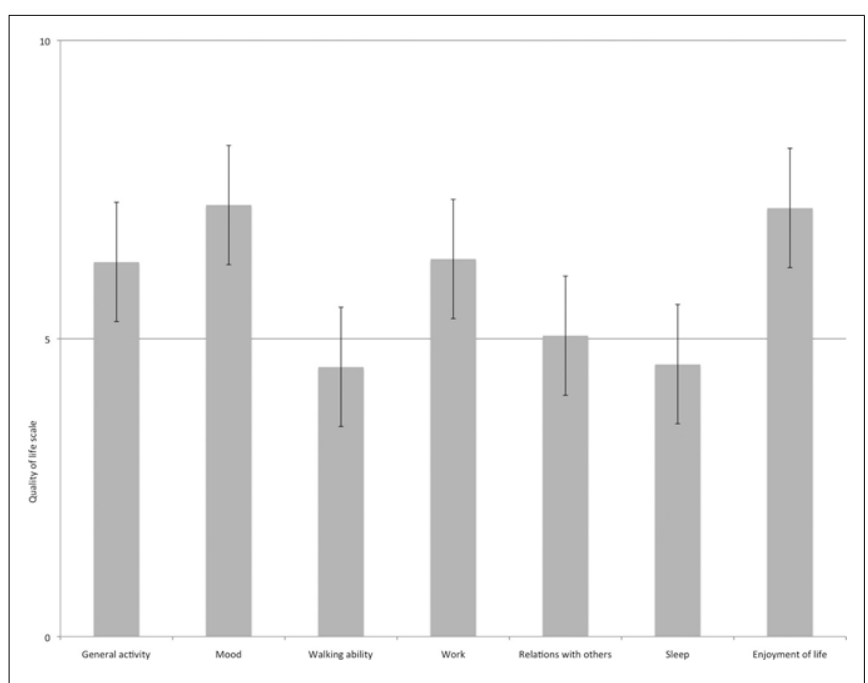

FIGURE 3 | Pain interference with general activity, mood, walking ability, work, social relations, sleep, and enjoyment of life in an 11-point scale of the Brief Pain Inventory $(0=$ "no interference" e $\mathbf{1 0}=$ "interferes completely"). The horizontal line defines the median point of the scale (5).

mood was $7.2 \pm 2.8$, with walking was $4.5 \pm 3.8$, with work was $6.3 \pm 3.6$, with relations with others was $5.1 \pm 4.2$, with sleep was $4.6 \pm 3.8$ and with enjoyment of life was $7.2 \pm 3.2$ (Figure 3).

The patients' subjective descriptions of their pain using the words from the short version of the MPQ are detailed in Table 3. The sensory and affective scores of the MPQ were, respectively, 12.1 and 6.2; the total score was 22.3. Actual pain intensity in a six-point scale was $1.6 \pm 0.4$. All the patients used more than one word to describe their pain, and always used words from both categories, sensory and affective. The words most frequently used were "heavy" and "tiring-exhausting" in $91.4 \%$ of the patients, and "hot-burning" in $58.8 \%$ of the cases.

\section{DISCUSSION}

The prevalence of pain found in this study, 34\%, is in accordance with the range of results from other European countries (Clifford and Trotter, 1984; Vermote et al., 1986; Stenager et al., 1991). However, there is some variability that might be related to differences/heterogeneity between the studied populations, and/or variation in the methodologies used. For example, studies differ in the sources from which the samples were drawn (e.g., hospital, ward, clinic, community, MS organizations) as well as in the sampling methodologies used (e.g., random, convenience). In our study, pain characterization by the patients was done reporting to pain experienced in the week previous to the study session, avoiding memory and other cognitive biases and/or symptom fluctuation that could interfere with the results. Headache was included in the evaluation of pain location (which was not reported in other studies; Clifford and Trotter, 1984; Stenager et al., 1991), because there is evidence that it may be secondary to MS disease processes (Archibald et al., 1994). However, including patients that reported pain in the week before the study visit may have, on the other hand, introduced a bias on the prevalence of episodic pains like headache. The MS diagnostic criteria used in the present study, from the International Panel on the Diagnosis of Multiple Sclerosis of 2001, and revised in 2005 (Polman et al., 2005), may have also had an impact in our results, especially when compared with older studies, because the actual criteria have more sensitivity and specificity, and allow earlier disease diagnosis (Poser et al., 1983; CHAMPS Study Group, 2002; Dalton et al., 2002). In this way, we strongly believe patients with other diseases besides MS were excluded, a possible confounder of the prevalence figures.

The finding that the head, back, and upper limbs were the most common pain sites in our Portuguese MS population is consistent with other studies (Ehde et al., 2006). Similarly to Ehde et al. (2006) and Stenager et al.'s (1991) results, we have not found an association neither between pain intensity and MS duration (as measured by the time since the beginning of the symptoms, and not by the time since initial diagnosis) nor between pain intensity and functional impairment (EDSS score). Results in the literature are dissimilar, maybe reflecting once more methodological issues or population discrepancies. The dissimilarity may additionally reflect heterogeneity of pain mechanisms in MS: it is known that just one lesion in a key area of the central nervous system can cause a neuropathic pain syndrome, implying that this type of pain can appear at any stage of MS. On the other hand, the higher the functional impairment during disease progression, the more likely is pain from the nociceptive type to appear, secondary for example to spasticity and mobility problems, making these associations difficult, and probably even not useful to make.

Regarding BPI results, only $10 \%$ of the patients were taking drugs for neuropathic pain treatment for pain relief. On the other hand, patient pain descriptions' from the MPQ included the word "hot-burning" in $58.8 \%$ of the cases, a word commonly used in the report of central pain. Considering these results and the fact that neuropathic pain is referred in the literature as the most common pain syndrome in MS (Fischer et al., 1999; Osterberg et al., 2005), our patients are probably being undertreated for their pain syndromes.

Pain interfered meaningfully with general activity, mood, work, relations with others, and enjoyment of life, that is, five of the seven parameters used in the QoL assessment. According to Daut et al. (1983), pain intensity or interference in QoL can be considered meaningful above the median point 5 of the BPI scales. In view of this, maximum pain intensity was meaningful in our study (Figure 2), as well as pain interference with general activity, mood, work, relations with others, and enjoyment of life (Figure 3). To our knowledge, only in a study by Ehde et al. (2006) was the BPI used as a pain and QoL measure in MS patients. The average level of overall pain interference with QoL was significantly higher in our study ( $t$ test, $p<0.001$ ), further supporting the idea that pain has a major impact in the QoL of our Portuguese sample of MS patients.

Pain revealed to be an important symptom in this sample of 85 MS patients, not just for the reason of the high prevalence found - in line with results from other European countries - but also because of the major interference of pain with the QoL of these patients. Given that the majority of patients were young adults and had a low education level and literacy [corroborated by the Organization for Economic Co-operation and Development (OECD); www.oecd.org/dataoecd/44/35/14735458.pdf, accessed 
in June 6, 2009], this may have consequences in an individual perspective, possibly making it more difficult for patients to understand MS diagnosis, prognosis, impact in daily life, and medication regimes, and, consequently, reducing compliance. In light of our results, we believe that an extra effort should be made to better identify MS patients with clinically meaningful pain syndromes in

\section{REFERENCES}

Alonso, A., and Hernán, M. A. (2008). Temporal trends in the incidence of multiple sclerosis: a systematic review. Neurology 71, 129-135.

Archibald, C. J., McGrath, P. J., Ritvo, P. G., Fisk, J. D., Bhan, V., Maxner, C. E., and Murray, T. J. (1994). Pain prevalence, severity and impact in a clinic sample of multiple sclerosis patients. Pain 58, 89-93.

Boneschi, F. M., Colombo, B., Annovazzi, P., Martinelli, V., Bernasconi, L., Solaro, C., and Comi, G. (2008). Lifetime and actual prevalence of pain and headache in multiple sclerosis. Mult. Scler. 14, 514-521.

CHAMPS Study Group. (2002). MRI predictors of early conversion to clinically definite MS in the CHAMPS placebo group. Neurology 59, 998-1005.

Clifford, D. B., and Trotter, J. L. (1984). Pain in multiple sclerosis. Arch. Neurol. 41, 1270-1272.

Dalton, C. M., Brex, P. A., Miszkiel, K. A., Hickman, S. J., MacManus, D. G., Plant, G. T., Thompson, A. J., and Miller, D. H. (2002). Application of the new McDonald criteria to patients with clinically isolated syndromes suggestive of multiple sclerosis. Ann. Neurol.52, 47-53.

Daut, R. L., Cleeland, C. S., and Flanery, R. C. (1983). Development of the Wisconsin Brief Pain Questionnaire to assess pain in cancer and other diseases. Pain 17, 197-210.

De Sá, J., Paulos, A., Mendes, H., Becho, J., Marques, J., and Roxo, J. (2006). The prevalence of multiple sclerosis in the District of Santarém, Portugal. J. Neurol. 253, 914-918.
Ehde, D. M., Osborne, T. L., Hanley, M. A., Jensen, M. P., and Kraft, G. H. (2006). The scope and nature of pain in persons with multiple sclerosis. Mult. Scler. 12, 629-638.

Fischer, J. S., LaRocca, N. G., Miller, D. M., Ritvo, P. G., Andrews, H., and Paty, D. (1999). Recent developments in the assessment of quality of life in multiple sclerosis (MS). Mult. Scler. 5, 251-259.

Hadjimichael, O., Kerns, R. D., Rizzo, M. A., Cutter, G., and Vollmer, T. (2007). Persistent pain and uncomfortable sensations in persons with multiple sclerosis. Pain 127, 35-41.

Kurtzke, J. F. (1980). Geographical distribution of multiple sclerosis. An update with special reference to Europe and the Mediterranean region. Acta Neurol. Scand. 62, 6580.

Kurtzke, J. F. (1983). Rating neurologic impairment in multiple sclerosis: an expanded disability status scale (EDSS). Neurology 33, 1444-1452.

Melzack, R. (1987). The short-form McGill Pain Questionnaire. Pain 30, 191-197.

Melzack, R. (2005). The McGill Pain Questionnaire: from description to measurement. Anesthesiology 103, 199-202.

Osborne, T. L., Raichle, K. A., Jensen, M. P., Ehde, D. M., and Kraft, G. (2006). The reliability and validity of pain interference measures in persons with multiple sclerosis. J. Pain Symptom Manage. 32, 217-229.

Osterberg, A., Boivie, J., and Thuomas, K. A. (2005). Central pain in multiple sclerosis: prevalence and clinical characteristics. Eur. J. Pain 9, 531-542.

order to properly manage and treat these patients attenuating the devastating consequences of chronic pain.

\section{ACKNOWLEDGMENTS}

Portuguese Foundation for Science and Technology (SFRH/BD/ 27411/2006).

Piwko, C., Desjardins, O. B., Bereza, B. G., Machado, M., Jaszewski, B. Freedman, M. S., Einarson, T. R., and Iskedjian, M. (2007). Pain due to multiple sclerosis: analysis of the prevalence and economic burden in Canada. Pain Res. Manag. 12, 259-265.

Polman, C. H., Reingold, S. C., Edan, G., Filippi, M., Hartung, H. P., Kappos, L., Lublin, F. D., Metz, L. M., McFarland, H. F., O'Connor, P. W., Sandberg-Wollheim, M., Thompson, A. J., Weinshenker, B. G. and Wolinsky, J. S. (2005). Diagnostic criteria for multiple sclerosis: 2005 revisions to the "McDonald Criteria”. Ann. Neurol. 58, 840-846.

Poser, C. M., Paty, D. W., Scheinberg, L., McDonald, W. I., Davis, F. A., Ebers, G. C., Johnson, K. P., Sibley, W. A., Silberberg, D. H., and Tourtellotte, W. W. (1983). New diagnostic criteria for multiple sclerosis: guidelines for research protocols. Ann. Neurol. 13, 227-231.

Rosati, G. (2001). The prevalence of multiple sclerosis in the world: an update. Neurol. Sci. 22, 117-139.

Solaro, C., Brichetto, G., Amato, M. P., Cocco, E., Colombo, B., D’Aleo, G., Gasperini, C., Ghezzi, A., Martinelli, V., Milanese, C., Patti, F., Trojano, M., Verdun, E., Mancardi, G. L., and PaIMS Study Group. (2004). The prevalence of pain in multiple sclerosis. A multicenter cross-sectional study. Neurology 63 919-921.

Stenager, E., Knudsen, L., and Jensen, K. (1991). Acute and chronic pain syndromes in multiple sclerosis. Acto Neurol. Scand. 84, 197-200.
Svendsen, K. B., Jensen, T. S., Hansen, H. J., and Bach, F. W. (2005). Sensory function and quality of life in patients with multiple sclerosis and pain. Pain 114, 473-481.

Tourtellote, W.-W., and Baumhefner, W. W. (1983). "Comprehensive management of multiple sclerosis," in Multiple Sclerosis, eds J. F. Hallpike, C. W. M. Adams, and W.-W. Tourtellote (Philadelphia: Lippincott Williams \& Wilkins), 513-578.

Vermote, R., Ketelaer, P., and Carton, H. (1986). Pain in multiple sclerosis patients. A prospective study using the McGill Pain Questionnaire. Clin. Neurol. Neurosurg. 88, 87-93.

Conflict of Interest Statement: The authors declare that the research was conducted in the absence of any commercial or financial relationships that could be construed as a potential conflict of interest.

Received: 14 September 2010; accepted: 16 March 2011; published online: 31 March 2011.

Citation: Seixas D, Sá MJ, Galhardo V, Guimarães J and Lima D (2011) Pain in Portuguese patients with multiple sclerosis. Front. Neur. 2:20. doi: 10.3389/fneur.2011.00020

This article was submitted to Frontiers in Multiple Sclerosis and Neuroimmunology, a specialty of Frontiers in Neurology. Copyright (c) 2011 Seixas, Sá, Galhardo, Guimarães and Lima. This is an openaccess article subject to a non-exclusive license between the authors and Frontiers Media SA, which permits use, distribution and reproduction in other forums, provided the original authors and source are credited and other Frontiers conditions are complied with. 